\title{
Development of a core outcome set (COS) for studies relating to awareness and clinical management of reduced fetal movement: study protocol
}

\author{
Dexter J. L. Hayes ${ }^{1 *}$ (D), Declan Devane ${ }^{2}$, Jo C. Dumville ${ }^{3}$, Valerie Smith ${ }^{4}$, Tanya Walsh ${ }^{5}$ and Alexander E. P. Heazell ${ }^{1}$
}

\begin{abstract}
Background: Concerns regarding reduced fetal movements (RFM) are reported in 5-15\% of pregnancies, and RFM are associated with adverse pregnancy outcomes including fetal growth restriction and stillbirth. Studies have aimed to improve pregnancy outcomes by evaluating interventions to raise awareness of RFM in pregnancy, such as kick counting, evaluating interventions for the clinical management of RFM, or both. However, there is not currently a core outcome set (COS) for studies of RFM. This study aims to create a COS for use in research studies that aim to raise awareness of RFM and/or evaluate interventions for the clinical management of RFM.

Methods: A systematic review will be conducted, to identify outcomes used in randomised and non-randomised studies with control groups that aimed to raise awareness of RFM (for example by using mindfulness techniques, fetal movement counting, or other tools such as leaflets or mobile phone applications) and/or that evaluated the clinical management of RFM.

An international Delphi consensus will then be used whereby stakeholders will rate the importance of the outcomes identified in the systematic review in (i) awareness and (ii) clinical management studies. The preliminary lists of outcomes will be discussed at a consensus meeting where one final COS for awareness and management, or two discrete COS (one for awareness and one for management), will be agreed upon.
\end{abstract}

Discussion: A well-developed COS will provide researchers with the minimum set of outcomes that should be measured and reported in studies that aim to quantify the effects of interventions.

Keywords: Core outcome set, Pregnancy, Reduced fetal movement

\section{Background}

\section{Reduced fetal movement and adverse pregnancy} outcome

Reduced fetal movements (RFM) are usually defined as a subjective decrease or change in a baby's typical pattern of movements in utero [1]; current guidance in the UK

\footnotetext{
*Correspondence: dexter.hayes@manchester.ac.uk

'Tommy's Stillbirth Research Centre, School of Medical Sciences, Division of Developmental Biology and Medicine, Faculty of Biology, Medicine, and Health, University of Manchester, Manchester, UK

Full list of author information is available at the end of the article
}

and Australia is for anyone pregnant to contact a midwife or maternity unit if their baby is moving less than usual or not at all $[2,3]$. Most pregnant people become aware of fetal movements by $18-20$ weeks' gestation, the pattern of their baby's movements, and the time of day that the baby moves the most by 28 weeks' gestation [4]. Awareness of fetal activity is recognised as one component of maternal-fetal attachment [5].

Maternal concern regarding RFM leads to a presentation at the hospital in $5-15 \%$ of pregnancies [6]. Around

(c) The Author(s). 2021 Open Access This article is licensed under a Creative Commons Attribution 4.0 International License, which permits use, sharing, adaptation, distribution and reproduction in any medium or format, as long as you give appropriate credit to the original author(s) and the source, provide a link to the Creative Commons licence, and indicate if changes were made. The images or other third party material in this article are included in the article's Creative Commons licence, unless indicated otherwise in a credit line to the material. If material is not included in the article's Creative Commons licence and your intended use is not permitted by statutory regulation or exceeds the permitted use, you will need to obtain permission directly from the copyright holder. To view a copy of this licence, visit http://creativecommons.org/licenses/by/4.0/ The Creative Commons Public Domain Dedication waiver (http://creativecommons.org/publicdomain/zero/1.0/) applies to the data made available in this article, unless otherwise stated in a credit line to the data. 
$70 \%$ of these pregnancies have a normal outcome [7-9], but observational studies have recurrently demonstrated that RFM are associated with adverse pregnancy outcomes, including fetal growth restriction (FGR) and stillbirth, supporting the potential for a common aetiology $[10,11]$. Case-control studies have consistently demonstrated an association between reduced frequency and strength of fetal movements and stillbirth after 28 weeks' gestation [12-14]; this effect has also been seen in lowincome settings [15]. It is thought that RFM may be an attempt by the fetus to conserve energy and oxygen consumption in cases of insufficient nutrient transfer and hypoxia, which in turn may be caused by placental insufficiency or other fetal stressors [16-18].

\section{Studies of awareness of or interventions for reduced fetal movement}

Studies have aimed to improve pregnancy outcomes by evaluating interventions that raise maternal and/or clinical awareness of RFM, such as mindfulness or kick counting $[19,20]$, and/or by evaluating clinical management interventions, for example, interventions comprised of further monitoring and/or clinical testing such as cardiotocography (CTG) or ultrasound (US) to identify whether RFM is an indicator of an underlying condition that may warrant further clinical intervention or even expedited birth $[21,22]$.

Despite the association between RFM, stillbirth and FGR, a core outcome set (COS) for studies evaluating interventions that aim to raise awareness of RFM and/or studies evaluating the clinical management of RFM does not currently exist. This means that studies often measure and report different outcomes and employ different definitions for these outcomes, hindering meta-analysis of studies. A COS describes a standardised set of outcomes that should be measured and reported in all studies in a specific area as a minimum [23]; COS are currently in use across several healthcare fields, including maternity care [24-26]. It is anticipated that developing a COS will ensure that the most important and relevant outcomes, as agreed by stakeholder consensus, are measured, thus optimising the synthesis of individual studies. This will further facilitate data synthesis and interpretation of the evidence based on prioritised outcomes.

\section{Methods}

\section{Aim}

This protocol describes the development of a COS for measurement and reporting in studies that aim to raise awareness of RFM and/or evaluate the clinical management of RFM. Development and adoption of this COS will ensure consistent and relevant outcome measurement and reporting in studies for raising awareness and/ or evaluating the clinical management of RFM, which may lead to more robust results, improved wellbeing in pregnancy, and may also be applicable in clinical practice.

This COS will apply to controlled randomised and non-randomised study designs, as described in Table 1.

\section{Objectives}

1. To systematically review the outcomes included in intervention studies for raising awareness of RFM and/or evaluating its clinical management

2. To develop a consensus on a preliminary COS using these outcomes via the Delphi survey

3. To develop a final COS for use in all future intervention studies aimed at raising awareness of RFM and/or evaluating the clinical management of RFM, via an international consensus meeting with key stakeholders

4. To disseminate and promote the use of the COS

\section{Design}

The COS development project was registered with the Core Outcome Measures in Effectiveness Trials (COMET) initiative (http://comet-initiative.org/Studies/ Details/928) on the 24th of September 2020.

This protocol was developed in accordance with the COMET handbook [23]. A study steering committee will be established comprised of at least one representative from the following relevant stakeholder groups: researchers, clinicians, and patients (described in more detail later). We will include at least one representative from low- or middle-income countries on the steering committee.

\section{Stage 1: Systematic review}

A systematic review of the literature will be conducted to identify outcomes measured in studies of interventions where the intervention is designed to raise awareness of RFM and/or evaluate the clinical management of RFM.

The target population is women with non-anomalous singleton pregnancies after 28 weeks' gestation; this threshold has been chosen over other definitions of stillbirth in order to facilitate international comparisons [27]. We will not include studies of multiple pregnancies because observational data about the association between RFM and adverse outcome in this group is less clear.

We will include controlled randomised and nonrandomised studies with clearly reported mechanisms of group formation, clearly defined inclusion criteria, and clearly described methods of ascertainment of eligible patients and their recruitment. Studies will be included 
Table 1 Study inclusion criteria for the systematic review

\begin{tabular}{|c|c|}
\hline Population & Singleton pregnancies presenting at least once in a maternity care setting after 28 weeks' gestation \\
\hline Intervention & Any intervention aimed at raising awareness of RFM and/or evaluating the clinical management of RFM \\
\hline Comparator & Any other intervention described above or no intervention \\
\hline Outcome & Any maternal or fetal outcomes \\
\hline $\begin{array}{l}\text { Study } \\
\text { design }\end{array}$ & $\begin{array}{l}\text { Controlled randomised and non-randomised studies with clearly reported mechanism of group formation, clearly defined inclusion cri- } \\
\text { teria, and described methods of ascertainment of eligible patients and their recruitment }\end{array}$ \\
\hline
\end{tabular}

regardless of their publication status and language of publication.

Pregnancy, labour, and birth outcomes will be extracted with their corresponding definitions where possible. Outcomes will be grouped as maternal or neonatal, and different definitions of the same outcome will be grouped into single outcome measures. This will be facilitated by discussions between members of the study team. This final list of outcomes will be used in stage two.

We will search the following databases: MEDLINE, MEDLINE (In-Process and Other Non-Indexed Citations), Embase, EBSCO CINAHL Plus, the Cochrane Central Register of Controlled Trials (CENTRAL), the Cochrane Pregnancy and Childbirth's Trials Register, and the Cochrane Database of Systematic Reviews. Other trial registries such as clinicaltrials.gov, WHO ICTRP, and the EU clinical trials register will also be searched, as well as databases such as OpenGrey (www. opengrey.eu), Joanna Briggs Institute (www.joannabriggs. edu.au), and the National Institute for Health and Clinical Excellence website (NICE; www.nice.org.uk) to find unpublished studies. Reference lists of included papers will be reviewed for additional studies. The search strategy for this COS is provided as an additional file.

Studies identified by the literature searches will be independently screened for inclusion by two study authors using our study inclusion criteria (Table 1). Disagreements will be resolved by consulting a third author. The following data will be extracted from included studies: study aim, location, population, setting, description of the intervention and comparator, and outcomes reported in the study.

\section{Stage 2: Online international Delphi survey}

A sequential three-round electronic international Delphi study will be conducted using REDCap v10.1.2 including key stakeholders to produce a preliminary COS. Each round will remain open for at least 14 days, and a reminder email will be sent out three working days before closure. Data from each round will be analysed and presented to participants in the next round (described in more detail below). Attrition rates for each round will also be assessed. All participants' contact information will remain confidential.
The Delphi survey and following consensus meeting will allow the possibility of producing either one COS (for all studies relating to raising awareness and/or evaluating the clinical management of RFM) or two COS (one for studies raising awareness of RFM and another for studies evaluating the clinical management of RFM). This will be dependent on whether there is significant overlap or similarity in the final outcomes in the two lists. This follows the precedent set by other COS in maternity care that have started by running two surveys simultaneously and then voted on whether one COS should be produced $[26,28]$.

\section{Participants}

We will invite people from all stakeholder groups, aiming for at least 15 people from each of the following three groups to ensure adequate representation. Eligible participants will be (1) researchers, research funders, and policymakers who are actively involved in work related to RFM; (2) clinicians (midwives, obstetricians, neonatologists, GPs/family physicians) with experience of caring for women with RFM; and (3) anyone who is or who has been pregnant and their partners if applicable. We will recruit participants through professional organisations, electronic discussion lists, and patient organisations or charities. As with the formation of the steering group, we will ensure that participants from low-income countries are represented. Authors of all included studies will be invited to participate; we will also use snowball sampling, whereby we will request participants to forward the survey to others who they consider eligible to participate.

Participants of the Delphi survey will receive all information regarding the study as part of the invitation email. Consent to take part in the survey will be ensured by requiring participants to click an 'I agree to take part' box before gaining access to the survey. All personal data of participants will only be accessible to members of the research team and any response to the survey will be confidential. Participants will have the right to withdraw at any point.

\section{Round 1}

Round 1 will collect demographic data including nationality, age, stakeholder group, and role. Participants will 
be presented with a list of all outcomes identified from the systematic review and will be asked to rate the importance of each using a 9-point Likert scale. On this scale, a score of 1-3 indicates limited importance, 4-6 signifies importance, and 7-9 is used for critically important outcomes. Participants will also be prompted to add additional outcomes that they feel are important but are not included in the preliminary list. Suggested outcomes will be included in round 2 if they are mentioned by at least two participants. All outcomes from round 1 will be forwarded to round 2 .

Feedback will be provided to all who participated in round 1 in the form of descriptive statistics and graphical representations for ease of interpretation. For each outcome, participants will receive their scores from the first round and a graphical representation of the percentages of each group who voted for each score for each outcome. All feedback provided to participants will be anonymised.

Standardised consensus criteria will be applied to the results from this round, which will be used through all three rounds to reach the preliminary list of outcomes to be included (Table 2). Outcomes that are not scored by participants will not be included in analyses or consensus definitions.

\section{Round 2}

Participants will be presented with their scores from the previous round as well as feedback given in the same way as described in round 1. All participants who completed the first round will be asked to re-score all outcomes using the same 9-point Likert scale, including any additional suggested outcomes from round 1, in light of their and others' ratings.

In round 2, outcomes will be presented in two lists. Participants will be asked to rate the outcomes in each list according to how important they think each outcome is for measuring and reporting in studies of (i) interventions for raising awareness of RFM and in studies of (ii) interventions for the clinical management of RFM. The ratings for the two separate lists will be reviewed and analysed separately.

Outcomes will be included in round 3 if they are rated as 'consensus in' or 'no consensus' using the consensus

Table 2 Consensus criteria for outcomes

\begin{tabular}{ll}
\hline Definition & Criteria \\
\hline $\begin{array}{l}\text { Consensus } \\
\text { in }\end{array}$ & $\begin{array}{l}\text { Scored as 7-9 by } 70 \% \text { or more of all participants, } \\
\text { including at least one from each stakeholder group, and } \\
\text { as 1-3 by less than 15\% of participants }\end{array}$ \\
$\begin{array}{ll}\text { Consensus } \\
\text { out }\end{array}$ & $\begin{array}{l}\text { Scored as 1-3 by over 70\% of participants and as 7-9 by } \\
\text { less than 15\% }\end{array}$ \\
$\begin{array}{ll}\text { No } \\
\text { consensus }\end{array}$ & Any other combination of scores \\
\hline
\end{tabular}

criteria in Table 2; those rated as 'consensus out' will be removed. Outcomes will be removed from lists (i) and (ii) of the survey individually based on their ratings in each list. We will also assess the rates of attrition from each round and whether participants change their scores based on the feedback they receive.

\section{Round 3}

Round 3 will only include participants who completed round 2. Participants will again be provided with feedback and asked to re-rate the outcomes retained from round 2 in the same way as in round 2, in two separate lists for (i) RFM awareness studies and (ii) RFM clinical management studies using the 9-point Likert scale. The consensus criteria will again be used to determine which outcomes are retained in each distinct outcome list following this round and forwarded to the consensus meeting. Those defined as 'consensus out' and 'no consensus' will be removed. Round 3 will include a question asking if participants are willing to take part in the final consensus meeting and if they consent to being contacted.

\section{Stage 3: Consensus meeting}

A consensus meeting with an international panel representing views of all key stakeholders will be held to discuss, vote, and agree on the final RFM COS. This meeting will include a presentation of the findings of the round 3 Delphi, including the final list of outcomes by category (i.e. awareness and clinical management) and how they were voted for by each stakeholder group. This will be followed by a timed discussion and a vote on each outcome for each list. Outcomes will be included if voted for by at least $70 \%$ of participants, including at least one from each stakeholder group.

The final COS for (i) interventions for raising awareness of RFM and (ii) interventions for the clinical management of RFM will then be compared. If the outcomes included in these two COS lists are largely the same, then a majority vote will be held on whether to combine the lists to produce one COS for studies of both the awareness and clinical management of RFM. If the outcomes in the lists are different, then two separate COS will be created.

The consensus panel will be comprised of at least three representatives from each stakeholder group described earlier. The meeting will be held in English and group facilitators will be used for discussions. Due to the COVID-19 pandemic and in order to facilitate international attendance, we are planning that this meeting will be online.

\section{Stage 4: Dissemination}

The final COS will be published in an open-access journal. After publication, it will be made available through 
the COMET and CoRe Outcomes in Women's and Newborn health (CROWN) databases. In addition, we plan to disseminate the COS at national and international conferences and through relevant professional and patient organisations. We will share the COS with clinical trial registries and relevant consumer groups such as maternity service users and will ask all participants to share as they see appropriate.

\section{Discussion}

Research into the management of RFM was identified by the Stillbirth Priority Setting Partnership (PSP) which prioritised the question 'Which investigations identify a fetus which is at risk of stillbirth after a mother believes she has experienced reduced fetal movements?' [29]. The PSP had extensive input from researchers, clinicians, stakeholder groups, and bereaved parents. However, there is currently no published COS for studies that evaluate interventions to raise awareness of RFM and/or for the clinical management of RFM. A welldeveloped COS for research studies that aim to quantify the effects of these interventions will provide researchers with a minimum set of outcomes that should be recorded, facilitating comparisons of interventions. We have taken steps to ensure that pregnant women and their views are adequately represented in this study and the final COS.

\section{Trial status}

Protocol version 1.1, 4th November 2021. Recruitment for the first round of the Delphi survey has begun.

\section{Abbreviations}

COS: Core outcome set; RFM: Reduced fetal movements

\section{Supplementary Information}

The online version contains supplementary material available at https://doi. org/10.1186/s13063-021-05839-9.

Additional file 1: Core Outcome Set-STAndardised Protocol Items: the COS-STAP Statement

\section{Acknowledgements}

We would like to thank Dr. Sanne Gordijn for providing feedback on this version of the study protocol.

\section{Authors' contributions}

$\mathrm{DH}, \mathrm{DD}, \mathrm{VS}$, and $\mathrm{AH}$ conceived the study. $\mathrm{DH}$ led the protocol development. All authors contributed to the study design and to the development of the protocol. All authors read and approved the final manuscript.

\section{Funding}

Financial support for this study was provided by Tommy's the baby charity. The funder had no role in the study design and will not be involved in the data collection, analyses, decision to publish, or preparation of the manuscript.

Availability of data and materials

Only the study authors will have access to the final dataset.

\section{Declarations}

Ethics approval and consent to participate

Ethical approval has been obtained from the University of Manchester Research Ethics Committee (Ref: 2021-11160-18073). Consent will be obtained from all participants by clicking an 'I consent to taking part' button before completing the survey.

Consent for publication

Not applicable.

\section{Competing interests}

The authors declare that they have no competing interests.

\section{Author details}

${ }^{1}$ Tommy's Stillbirth Research Centre, School of Medical Sciences, Division of Developmental Biology and Medicine, Faculty of Biology, Medicine, and Health, University of Manchester, Manchester, UK. ${ }^{2}$ HRB-Trials Methodology Research Network, School of Nursing and Midwifery, National University of Ireland, Galway, Ireland. ${ }^{3}$ Division of Nursing, Midwifery and Social Work, School of Health Sciences, Faculty of Biology, Medicine, and Health, University of Manchester, Manchester Academic Science Centre, Manchester, UK. ${ }^{4}$ School of Nursing \& Midwifery, Trinity College Dublin, The University of Dublin, Dublin, Ireland. ${ }^{5}$ School of Dentistry, University of Manchester,

Manchester, UK.

Received: 5 March 2021 Accepted: 17 November 2021

Published online: 09 December 2021

\section{References}

1. Jakes AD, Whybrow R, Spencer C, Chappell LC. Reduced fetal movements. BMJ. England; 2018;360:k570, DOl: https://doi.org/10.1136/bmj.k570.

2. RCOG. Reduced fetal movements Green-top Guideline No. 57 [Internet]. 2011. Available from: https://www.rcog.org.uk/globalassets/documents/ guidelines/gtg_57.pdf

3. Daly LM, Gardener G, Bowring V, Burton W, Chadha Y, Ellwood D, et al. Care of pregnant women with decreased fetal movements: update of a clinical practice guideline for Australia and New Zealand. Aust New Zeal J Obstet Gynaecol [Internet]. L.M. Daly, NHMRC Centre of Research Excellence in Stillbirth, Mater Research Institute, The University of Queensland, Brisbane, Australia. E-mail: lisa.daly@uq.edu.au; 2018;58:463-468. Available from: http:// obgyn.onlinelibrary.wiley.com/hub/journal/10.1111/(ISSN)1479-828X/

4. Raynes-Greenow CH, Gordon A, Li Q, Hyett JA. A cross-sectional study of maternal perception of fetal movements and antenatal advice in a general pregnant population, using a qualitative framework. BMC Pregnancy Childbirth [Internet]. BioMed Central; 2013 [cited 2020 Oct 7];13:1-8. Available from: https://link.springer.com/articles/10.1186/1471-2393-13-32, 13,1

5. Cranley MS. Development of a tool for the measurement of maternal attachment during pregnancy. Nurs Res [Internet]. 1981 [cited 2020 Nov 25]; 30:281-284. Available from: /record/1983-09386-001

6. Heazell AEP, Green M, Wright C, Flenady V, Frøen JF. Midwives' and obstetricians' knowledge and management of women presenting with decreased fetal movements. Acta Obstet Gynecol Scand. 2008;87(3):331-9. https://doi.org/10.1080/00016340801902034

7. Sinha D, Sharma A, Nallaswamy V, Jayagopal N, Bhatti N. Obstetric outcome in women complaining of reduced fetal movements. J Obstet Gynaecol (Lahore). 2007:27:41-43.

8. Dutton P, Warrander L, Bernatavicius G, Kroll J, Gaze D, Jones R, et al. Placentally-derived factors in women with reduced fetal movements and their relationship to pregnancy outcome. BJOG An Int J Obstet Gynaecol [Internet]. P. Dutton, University of Manchester, School of Medicine, Manchester, United Kingdom; 2012;119:e3. Available from: http://ovidsp. ovid.com/ovidweb.cgi?T=JS\&PAGE=reference\&D=emed $13 \& N E W S=N \& A N=$ 70780173

9. Higgins LE, Myers JE, Sibley CP, Johnstone ED, Heazell AEP. Antenatal placental assessment in the prediction of adverse pregnancy outcome after reduced fetal movement. PLoS One. United States; 2018;13:e0206533.

10. Ross C, Narain S, Sharma S. Perinatal outcomes in women who present with reduced fetal movements. BJOG An Int J Obstet Gynaecol. 2015. 
11. Levy M, Kovo M, Barda G, Gluck O, Koren L, Bar J, et al. Reduced fetal movements at term, low-risk pregnancies: is it associated with adverse pregnancy outcomes? Ten years of experience from a single tertiary center. Arch Gynecol Obstet [Internet]. M. Levy, Department of Obstetrics and Gynecology, The Edith Wolfson Medical Center (affiliated with Sackler Faculty of Medicine, Tel Aviv University), Tel Aviv, P.O Box 5, Holon 58100, Israel. E-mail: levmichal@gmail.com; 2020;301:987-993. Available from: https://www.springer.com/medicine/gynecology/journal/404

12. Stacey T, Thompson JMD, Mitchell EA, Ekeroma A, Zuccollo J, Mccowan LME. Maternal perception of fetal activity and late stillbirth risk: findings from the Auckland Stillbirth Study. Birth [Internet] 2011 [cited 2020 Feb 27]; 38:311-6. Available from: http://www.ncbi.nlm.nih.gov/pubmed/22112331, Maternal Perception of Fetal Activity and Late Stillbirth Risk: Findings from the Auckland Stillbirth Study

13. Heazell AEP, Budd J, Li M, Cronin R, Bradford B, McCowan LME, et al. Alterations in maternally perceived fetal movement and their association with late stillbirth: findings from the Midland and North of England stillbirth case-control study. BMJ Open [Internet]. BMJ Publishing Group; 2018 [cited 2019 Oct 1];8:e020031. Available from: http://www.ncbi.nlm.nih.gov/ pubmed/29982198

14. Heazell AEP, Warland J, Stacey T, Coomarasamy C, Budd J, Mitchell EA, et al. Stillbirth is associated with perceived alterations in fetal activity - findings from an international case control study. BMC Pregnancy Childbirth [Internet]. BioMed Central Ltd.; 2017 [cited 2020 Feb 27];17:369. Available from: http://www.ncbi.n/m.nih.gov/pubmed/29132322

15. Hayes D, Smyth $R$, Heazell A. Investigating the significance and current state of knowledge and practice of absent or reduced fetal movements in low and lower middle income countries: a scoping review. joghr.scholasticahq com [Internet]. 2019 [cited 2020 Nov 30];3:2019023. Available from: https:// doi.org/10.29392/joghr.3.e2019023, 3

16. Unterscheider J, Horgan R, O'Donoghue K, Greene R. Reduced fetal movements. Obstet Gynaecol. Wiley. 2009;11:245-51.

17. Warrander LK, Heazell AEP. Identifying placental dysfunction in women with reduced fetal movements can be used to predict patients at increased risk of pregnancy complications. Med Hypotheses. Churchill Livingstone. 2011; 76(1):17-20. https://doi.org/10.1016/j.mehy.2010.08.020.

18. Winje BA, Froen JF, Roald B, Kristensen NP. Placental pathology in pregnancies with maternally perceived decreased fetal movement - a population-based nested case-cohort study. PLoS One [Internet]. 2012;7. Available from: https://dx.plos.org/10.1371/journal.pone.0039259.

19. Grant A, Elbourne D, Valentin $L$, Alexander S. Routine formal fetal movement counting and risk of antepartum late death in normally formed singletons. Lancet (London, England). England. 1989;2:345-9.

20. Akselsson A, Lindgren H, Georgsson S, Pettersson K. Awareness of fetal movements and pregnancy outcomes among women born in Somalia and Sweden: a cluster-randomised controlled trial. 2020 [cited 2020 Aug 6]; Available from: https://www.diva-portal.org/smash/record.jsf?pid=diva2:142 7212

21. Norman JE, Heazell AEP, Rodriguez A, Weir CJ, Stock SJE, Calderwood CJ, et al. The AFFIRM study: can promoting awareness of fetal movements and focusing interventions reduce fetal mortality? A stepped-wedge cluster randomised trial. Am J Obstet Gynecol [Internet]. J.E. Norman, University of Edinburgh, Edinburgh, United Kingdom; 2018;218:S603. Available from: http://ovidsp.ovid.com/ovidweb.cgi?T=JS\&PAGE=reference\&D=emed1 9\&NEWS=N\&AN=620310333

22. Tveit JVH, Saastad E, Stray-Pedersen B, PE B, Flenady V, Fretts RC, et al. Reduction of late stillbirth with the introduction of fetal movement information and guidelines - a clinical quality improvement. BMC Pregnancy Childbirth [Internet]. BioMed Central; 2009;9:32. Available from: http:// bmcpregnancychildbirth.biomedcentral.com/articles/10.1186/1471-2393-9-32

23. Williamson PR, Altman DG, Bagley H, Barnes KL, Blazeby JM, Brookes ST, et al. The COMET handbook: version 1.0 [Internet]. Trials. BioMed Central Ltd.; 2017 [cited 2020 Oct 2]. p. 1-50. Available from: https://trialsjournal. biomedcentral.com/articles/10.1186/s13063-017-1978-4

24. Duffy J, Hooft J van't, Gale C. A protocol for developing, disseminating, and implementing a core outcome set for pre-eclampsia. Elsevier [Internet]. 2016 [cited 2020 Oct 22];Pregnancy. Available from: https://www. sciencedirect.com/science/article/pii/S2210778916300149?casa_token=KTtX3 gdSN3IAAAAA:12vmendOOw4KL7OV3P5OpF99EcrIKsT92BIG2zyrZ793 SvuiJCWilxNjuCNwAj3_RduFe8Y4Zw
25. Khalil A, Duffy J, Perry H, Ganzevoort W. Study protocol: developing, disseminating, and implementing a core outcome set for selective fetal growth restriction in monochorionic twin pregnancies. Trials [Internet]. 2019 [cited 2020 Oct 22]; Available from: https://doi.org/10.1186/s13063-018-31 53-y, 20, 1, 35

26. Healy P, Gordijn SJ, Ganzevoort W, Beune IM, Baschat A, Khalil A, et al. A Core Outcome Set for the prevention and treatment of fetal GROwth restriction: deVeloping Endpoints: the COSGROVE study. Am J Obstet Gynecol. 2019;221(339):e1-339.e10.

27. WHO | Stillbirths. WHO [Internet]. World Health Organization; 2016 [cited 2020 Nov 19]; Available from: http://www.who.int/maternal_child_a dolescent/epidemiology/stillbirth/en/

28. Egan AM, Bogdanet D, Griffin TP, Kgosidialwa O, Cervar-Zivkovic M, Dempsey $E_{\text {, et }}$ al. A core outcome set for studies of gestational diabetes mellitus prevention and treatment. Diabetologia [Internet]. Springer; 2020 [cited 2021 Oct 7];63:1120. Available from: /pmc/articles/PMC7228989/

29. Heazell AEP, Whitworth MK, Whitcombe J, Glover SW, Bevan C, Brewin J, et al. Research priorities for stillbirth: process overview and results from UK Stillbirth Priority Setting Partnership. Ultrasound Obs Gynecol [Internet]. John Wiley and Sons Ltd; 2015 [cited 2020 Oct 14];46:641-647. Available from: www.stillbirthpsp.org.uk

\section{Publisher's Note}

Springer Nature remains neutral with regard to jurisdictional claims in published maps and institutional affiliations.
Ready to submit your research? Choose BMC and benefit from:

- fast, convenient online submission

- thorough peer review by experienced researchers in your field

- rapid publication on acceptance

- support for research data, including large and complex data types

- gold Open Access which fosters wider collaboration and increased citations

- maximum visibility for your research: over $100 \mathrm{M}$ website views per year

At BMC, research is always in progress.

Learn more biomedcentral.com/submissions 